\title{
An Improved Formulation for Calorimetric Emittance Testing of Spacecraft Thermal Control Coatings
}

\author{
Lonny R. Kauder \\ NASA, Goddard Space Flight Center, Greenbelt, MD, 20771
}

\begin{abstract}
Spacecraft often really heavily on passive thermal control to maintain operating temperature. An important parameter in the spacecraft heat balance equation is the emittance of thermal control coatings as a function of coating temperature. One method for determining the emittance of spacecraft thermal control from elevated temperature to cryogenic temperatures relies on a calorimetric technique. The fundamental equation governing this test method can be found in numerous places in the literature and although it generally provides reasonable results, its formulation is based on a conceptual flaw that only becomes apparent when the sample temperature approaches the wall temperature during testing. This paper investigates the cause for this error and develops the correct formulation for calorimetric emittance testing. Experimental data will also be presented that illustrates the difference between the two formulations and the resulting difference in the calculated emittance.
\end{abstract}

\section{Introduction}

The determination of the total hemispherical emittance of spacecraft thermal control coatings at cryogenic temperatures is best accomplished utilizing a calorimetric technique. In this technique the coating of interest is typically applied to an aluminum substrate and suspended inside a liquid helium shroud under high vacuum and allowed to cool by thermal radiation to shroud walls. The emittance of the coatings surface can then be calculated with a knowledge of the mass of the substrate $(\mathrm{m})$, the specific heat as a function of temperature for the substrate $(\mathrm{Cp})$, the rate of cooling $(\Delta \mathrm{T} / \Delta \mathrm{t})$, the amount of heat lost through the thermocouple test leads $\left(\dot{Q}_{t c}\right)$, the amount of heat being lost due to the residual gas in the shroud ( $\left.\dot{Q}_{\text {gas }}\right)$, the area of the coating (a) and the difference between the sample temperature $(\mathrm{T})$ and the shroud wall temperature (Ts). The typical defining equation for the total hemispherical emittance can easily be found in numerous places in the literature ${ }^{1,2,4}$ and generally has a form similar to the equation below.

$$
\varepsilon_{h}=\frac{-m C p \frac{\Delta T}{\Delta t}-\dot{Q}_{t c}-\dot{Q}_{g a s}}{a \sigma\left(T^{4}-T s^{4}\right)}
$$


The equation (1) above generally gives good numerical results, however there are some situations for which this equation can give calculated emittance values greater than 1.0. This problem stems from the fact that, strictly speaking, this equation is incorrect from a theoretical standpoint. Consequently, under certain conditions, the equation (1) can give erroneous values.

\section{Theory}

The theoretical argument that leads to the above equation (1) generally begins with the heat balance equation for this problem ${ }^{1}$ :

$$
-m C p \frac{\Delta T}{\Delta t}=a \sigma \varepsilon_{h} T^{4}-a \sigma \varepsilon_{h} T s^{4}+\dot{Q}_{t c}+\dot{Q}_{g a s}
$$

Basically, the quantity of heat lost by the substrate is equal to the heat radiated by the coating minus the heat absorbed by the coating from the shroud plus the heat lost by conduction down the thermocouple wires plus the heat lost due to the residual gas in the chamber. The heat emitted by the shroud enclosure is of course black body radiation at the temperature of the shroud and some of this thermal energy is absorbed by the sample. Kirchoff's law ${ }^{5}$ is then employed to state that the hemispherical emittance of the sample at a given temperature is equal to its absorptance and consequently $\varepsilon_{h}$ is used in the second term of the equation (2) and solving for $\varepsilon_{h}$ leads to equation (1). The misinterpretation of Kirchoff's law is where the theoretical problem lies. The statement that the absorptance is equal to the hemispherical emittance at a given temperature is as course true, however it is only true if the radiating source is at the same temperature of the absorbing object. What Kirchoff's law really means is, that at a given temperature the absorptance is equal to the emittance and the sample will therefore absorb an amount of heat from a black body (at the same temperature as the sample) equal to the amount of heat it emits at that temperature. It will not necessarily absorb the same percentage of heat from a black body at any other temperature. For example; if the sample is at a temperature of $120^{\circ} \mathrm{K}$ and at that temperature it has an emittance of 0.70 , it will absorb $70 \%$ of the energy incident on it from a black body at $120^{\circ} \mathrm{K}$. It does not mean that it will absorb $70 \%$ of the energy incident on it from a black body at $30^{\circ} \mathrm{K}$ or $70 \%$ of the energy incident on it from a black body at $300^{\circ} \mathrm{K}$, only $70 \%$ of the energy incident on it from a black body at the same temperature it is, in this example $120^{\circ} \mathrm{K}$. To know how much energy the sample does absorb from a black body at any other temperature, it must be known what the emittance of the sample is at the temperature of the radiating source. Mathematically this is given by $a \sigma \varepsilon_{h}(\mathrm{Ts})$. Therefore, a better way to write the heat balance equation for the use in a calorimetric test is:

$$
-m C p \frac{\Delta T}{\Delta t}=a \sigma \varepsilon_{h}(T) T^{4}-a \sigma \varepsilon_{h}(T s) T s^{4}+\dot{Q}_{t c}+\dot{Q}_{g a s}
$$


Since $\varepsilon_{\mathrm{h}}(\mathrm{T}) \& \varepsilon_{\mathrm{h}}(\mathrm{Ts})$ are not necessarily equal, the emittance in the second term can not be factored and consequently the resulting defining equation for calorimetric emittance testing becomes:

$$
\varepsilon_{h}(T)=\frac{-m C p \frac{\Delta T}{\Delta t}-\dot{Q}_{t c}-\dot{Q}_{g a s}+a \sigma \varepsilon_{h}(T s) T s^{4}}{a \sigma T^{4}}
$$

The term governing the amount of heat absorbed by the sample from the shroud walls is now in the numerator instead of the denominator.

Generally, in calorimetric emittance testing, the sample temperature is not close to the shroud temperature and consequently the numerical value of the term representing the amount of heat absorbed by the sample from the shroud is small relative to the other terms in the equation. The resulting calculated emittance would therefore be numerically very similar no matter which equation is used. However, when the sample temperature approaches the shroud wall temperature the two equations can produce different values that can be significantly different. In the case where the sample temperature does approach the shroud wall temperature it is important to use the theoretically correct version of the heat balance equation in order to calculate the total hemispherical emittance of the sample correctly.

\section{Experiment Description}

To validate this new theoretical representation for the calculated total hemispherical emittance, an experiment was conducted at the Goddard Space Flight Center Calorimetric emittance test facility. An Aluminum substrate painted with Aeroglaze Z306 was used as the test article. One calorimetric emittance test was run using liquid helium to cool the shroud and a second test was run using liquid nitrogen to cool the shroud. The test article was not removed from the vacuum chamber and the vacuum was maintained during these two tests. The only major difference between the two tests was the temperature of the shroud. The test which used liquid helium to cool the shroud wall allowed for the determination of the emittance of the sample at liquid nitrogen temperature $\varepsilon_{h}(\mathrm{Ts})$ in equation (4). A comparison could then be made between the calculated emittance using each of the two heat balance equations for the test in which liquid nitrogen was used as the shroud wall coolant.

The test article consisted of an A1100 Aluminum substrate, $3.81 \mathrm{~cm}$ in diameter, $0.76 \mathrm{~cm}$ thick, and painted with Aeroglaze Z306 black paint 1.37mils thick. The black painted Aluminum substrate was suspended in the cryogenic shroud slightly off center to minimize any direct reflection from the shroud walls. A silicon diode temperature sensor embedded in the substrate provided temperature readings from $340^{\circ} \mathrm{K}$ to $50^{\circ} \mathrm{K}$. The electrical connections to the silicon diode (manganin wires), also supported the test article from the top of the cryogenic shroud. The figure below, gives a schematic representation of the experimental test set up. 


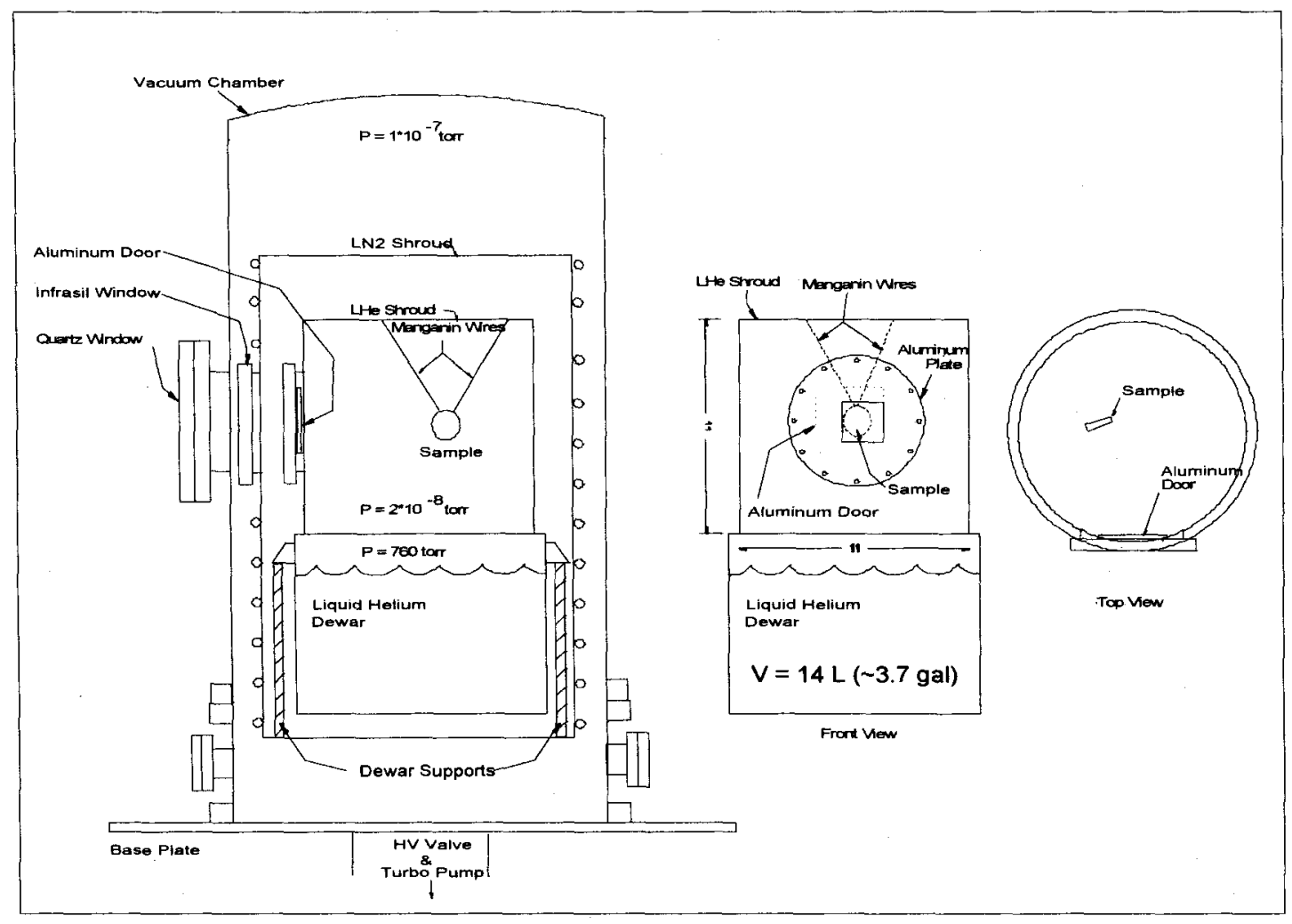

Fig. 1. Calorimetric Emittance test setup

\section{Test Procedure}

Testing was initiated by evacuating the chamber and cooling both inner and outer shrouds to their cryogenic operating temperatures. The sample was then heated through the vacuum chamber quartz \& infrasil windows via a Spectrolab X25 solar simulator. When a sample temperature of $340^{\circ} \mathrm{K}$ was reached, the solar simulator was switched off and the vacuum chamber window covered with an aluminum cap and the helium shroud aluminum door was closed. The sample was then allowed to cool by thermal radiation to the liquid helium shroud walls at $\approx 8^{\circ} \mathrm{K}$. Monitoring the cool down rate and having knowledge of the specific heat of the substrate as a function of temperature, the sample mass and total radiating area and also knowledge of the parasitic heat losses in the system, allowed the total hemispheric emittance to be calculated as a function of temperature. The first test was conducted with liquid Helium cooling the cryogenic shroud and the following equation (5) was used to calculate the total hemispherical emittance of the test article. 


$$
\varepsilon_{h k}=\frac{-m C p_{k} \frac{\Delta T_{k}}{\Delta t_{k}}-m_{z 306} C p_{z 306 k} \frac{\Delta T_{k}}{\Delta t_{k}}-Q_{t c k}-Q_{g a s_{k}}-Q_{s d k}+a \varepsilon_{h}(T s) \sigma T s_{k}{ }^{4}}{a \sigma T_{k}^{4}}
$$

Where:

$\sigma \quad$ : Stefan-Boltzman constant

$C p \quad$ : specific heat of substrate

$\Delta \mathrm{t} \quad$ : time increment

$C p_{2306}$ : specific heat of paint

$Q_{t c} \quad$ : residual gas heat loss

a : surface area of black paint

$T s \quad$ : temperature of shroud $m$ : mass of the Aluminum substrate

$\Delta T \quad$ : temperature increment

$m_{z 306}:$ mass of paint

$Q_{t c} \quad$ : manganin supports wires heat loss

$Q_{s d}$ : heat input from silicon diode

$T$ : temperature of substrate

The heat lost by the manganin support leads can be calculated from equations found in the literature ${ }^{3}$, or it can be derived from thermodynamic principles. The heat transfer by the residual gas in the chamber for a given sample temperature can be calculated by knowing the thermal conductivity of the residual gas. This equation can also be found in the literature ${ }^{6}$. The total heat flow from the test article can be schematically represented by the diagram below.

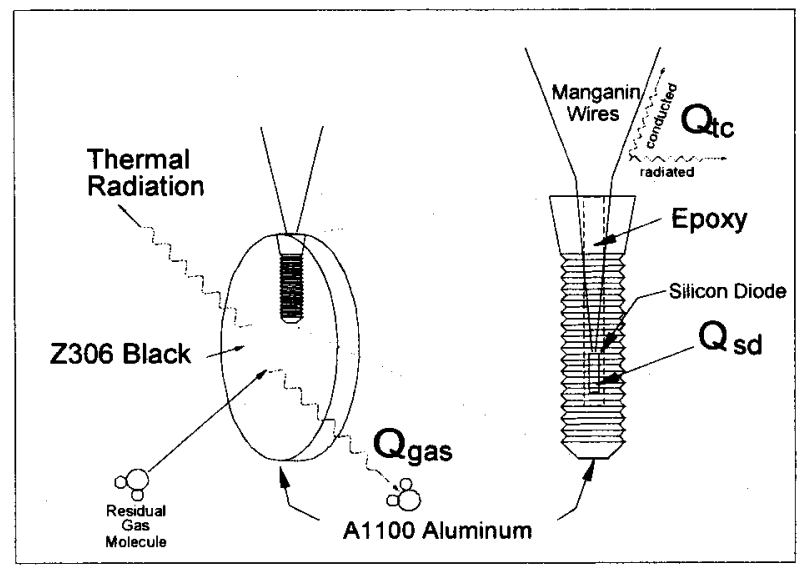

Fig. 2. Heat flow from test article

When the test article reached $50^{\circ} \mathrm{K}$, the first test was concluded. The liquid helium in the inner shroud was replaced with liquid nitrogen and the sample temperature was raised again to $340^{\circ} \mathrm{K}$. The inner shroud aluminum door was then closed and the test article allowed to cool by thermal radiation to the cryogenic shroud walls $\approx 77.5^{\circ} \mathrm{K}$. When the test article reached a temperature of $82^{\circ} \mathrm{K}$ the second test was concluded. The total hemispherical emittance was calculated both by equation (5) and for theoretical comparison by the following equation (6).

$$
\varepsilon_{h k}=\frac{-m C p_{k} \frac{\Delta T_{k}}{\Delta t_{k}}-m_{z 306} C p_{z 306 k} \frac{\Delta T_{k}}{\Delta t_{k}}-Q_{t c k}-Q_{g a s_{k}}-Q_{s d k}}{a \sigma\left(T_{k}^{4}-T s_{k}^{4}\right)}
$$




\section{Results and Conclusion}

The graph below shows the calculated total hemispherical emittance for each test. The emittance in the case where liquid nitrogen was used to cool the inner shroud wall was calculated by both the new (5) and old version (6) of the heat balance equation. Clearly when the old representation for the heat balance equation is used the calculated emittance tends to increase to infinity as the test article temperature approaches the shroud wall temperature. When the new representation is used instead, and the emittance of the sample at the shroud wall temperature is taken from the test data in which liquid helium was used to cool the shroud wall, the calculated emittance from the LN2 test matches that from the liquid helium test exactly. This gives experimental verification that the new formulation for the heat balance equation is correct.

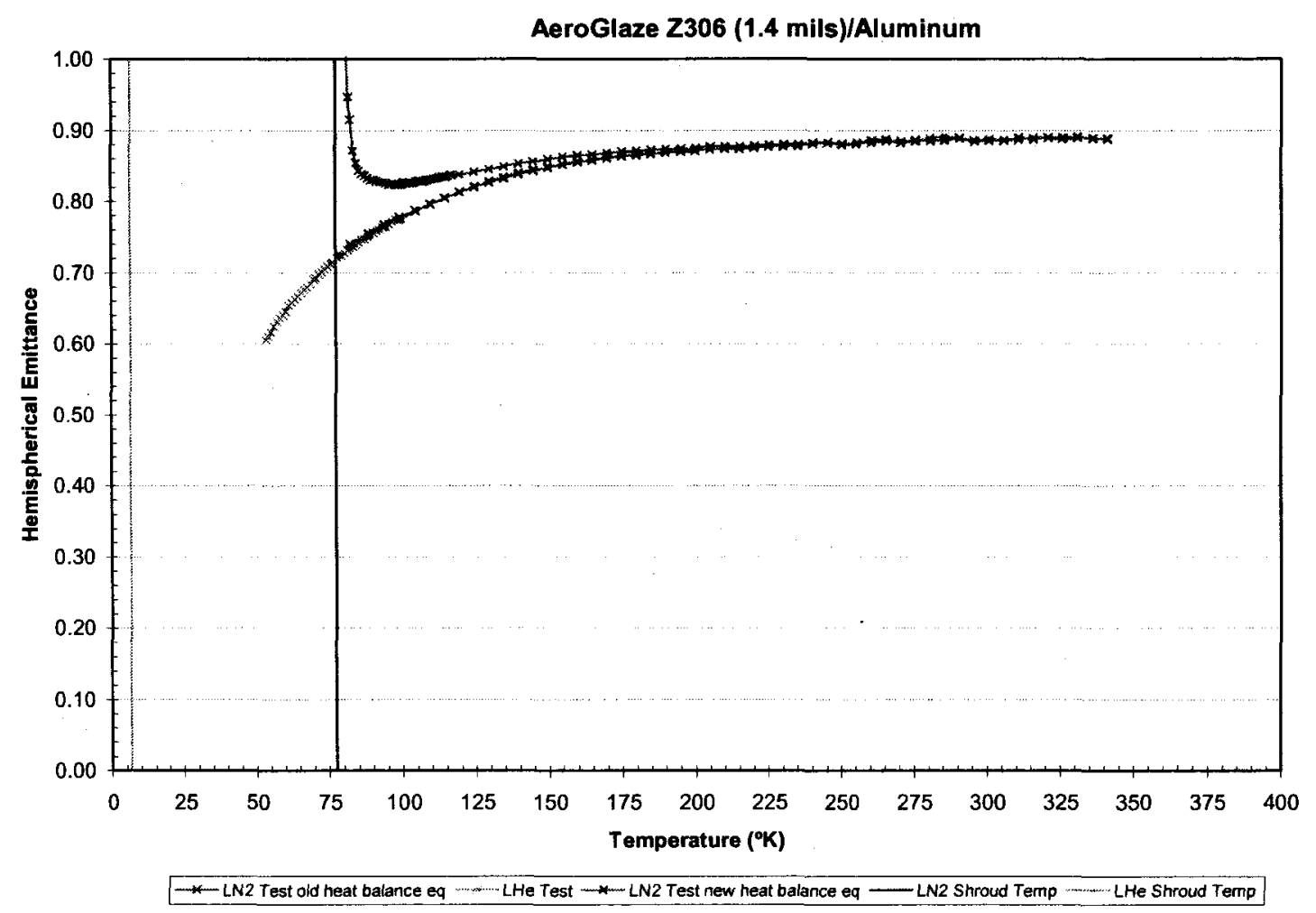

Fig.3. Comparison of both heat balance equations for Calorimetric test data

It can be seen that even at sample temperatures as much as $50^{\circ} \mathrm{K}$ above the wall temperature, the calculated emittance utilizing the old heat balance representation is $\approx 2 \%$ higher than the true value. It is therefore important to use the correct formulation in calculating the total hemispherical emittance when the sample temperature approaches the shroud wall temperature. 


\section{References}

${ }^{1 .}$ Edward A. Estalote and K.G. Ramanathan. Low-temperature emissivities of copper and aluminum. J. Opt. Soc. Am., Vol. 67, No. 1, January 1977.

2. Hidetoshi Masuda and Mitsuo Higano. Transient calorimetric technique for measuring total hemispherical emissivities of metals with rigorous evaluation of heat loss through thermocouple leads. J. Opt. Soc. Am. A, Vol. 2, No. 11, November 1985.

3. W.B. Fussell, J.J. Triolo, and J.H. Henninger. A Dynamic Thermal Vacuum Technique for Measuring The Solar Absorptance and Thermal Emittance of Spacecraft Coatings.

NASA Technical Note D-1716, March 1963.

4. D.P. Verret and K.G. Ramanathan. Total hemispherical emissivity of tungsten. J. Opt. Soc. Am., Vol 68, No. 9, September 1978.

5. Robert Siegel and John R. Howell. Thermal Radiation Heat Transfer Volume 1, The Blackbody, Electromagnetic Theory and Materials Properties. NASA sp-164 1968

${ }^{6}$ A. Roth. Vacuum Technology. North-Holland Publishing Company, New York, 1983. 